\title{
Corrigendum: Stimulus-specific combinatorial functionality of neuronal c-fos enhancers
}

Jae-Yeol Joo, Katie Schaukowitch, Lukas Farbiak, Gokhul Kilaru \& Tae-Kyung Kim

Nat. Neurosci. 19, 75-83 (2016); published online 23 November 2015; corrected after print 22 January 2016

In the version of this article initially published, it was stated that the $c$-fos gene-based TRAP mouse line described in ref. 42 utilized only the $c$-fos promoter region to drive CreER ${ }^{\mathrm{T} 2}$. In fact, that line was generated by CreER ${ }^{\mathrm{T} 2}$ knock-in to the endogenous $c$-fos locus. Accordingly, the sentence "Its induction mechanism and available reporter mouse lines are based exclusively on $c$-fos promoter activity" has been deleted from the Abstract, and the following sentences have been deleted from the third paragraph of the Discussion: "However, these mice utilize only the $c$-fos promoter region to induce the reporter fluorescent protein and do not include any of the $c$-fos enhancers we have characterized. On the basis of our findings, the promoter-only reporters might not faithfully recapitulate the expression characteristics of the endogenous $c$-fos gene in vivo triggered by sensory or pharmacological stimuli." The changes have been made in the HTML and PDF versions of the article.

\section{Erratum: Causal contribution of primate auditory cortex to auditory perceptual decision-making}

Joji Tsunada, Andrew S K Liu, Joshua I Gold \& Yale E Cohen

Nat. Neurosci. 19, 135-142 (2015); published online 14 December 2015; corrected after print 23 December 2015

In the version of this article initially published, the neurometric slope values in the bottom panel of Figure 4a were given as 1.6, 1.0., 1.0; the correct values are $0.8,0.5,0.5$. The $x$ axes in Figure $4 \mathrm{~b}$, right panel, were numbered 0 to 3 ; the correct range is 0 to 1.5 . The segments of the traces in Figure $6 \mathrm{~b}-\mathrm{d}$ with significant regression coefficients were gray; they should have been red. And the top segment of the bar for $0-10 \%$ in the bottom panel of Figure 7c was blue; it should have been pink. The errors have been corrected in the HTML and PDF versions of the article.

\section{Erratum: Schizophrenia and brain volume genetic covariation}

\section{P Alexander Arguello}

Nat. Neurosci. 19, 419 (2016); published online 23 February 2016; corrected after print 23 February 2016

In the version of this article initially published, the page number for the cross-referenced article was given as 414 instead of 420 and the author name was misspelled Frank instead of Franke. The errors have been corrected in the HTML and PDF versions of the article. 